\title{
Design and evaluation of a maize monitoring system for precision planting
}

\author{
Yanxin Yin ${ }^{1,2,3}$, Liping Chen ${ }^{1,2,3}$, Zhijun Meng ${ }^{1,2,3^{*}}$, Bin $\mathrm{Li}^{1,2,3}$, Changhai Luo ${ }^{1,2,3}$, \\ Weiqiang $\mathrm{Fu}^{1,2,3}$, Hebo $\mathrm{Mei}^{1,2,3}$, Wuchang Qin ${ }^{1,2,3}$ \\ (1. Beijing Research Center of Intelligent Equipment for Agriculture, Beijing Academy of Agriculture and Forestry Sciences, \\ Beijing 100089, China; 2. National Research Center of Intelligent Equipment for Agriculture, Beijing 100089, China; \\ 3. Beijing Key Laboratory of Intelligent Equipment Technology for Agriculture, Beijing 10089, China)
}

\begin{abstract}
To increase the accuracy and real-time performance of on-line assessment of maize planting, a CAN bus based maize monitoring system for precision planting was designed and tested both in laboratory and field. The system was mainly comprised of: (a) seeding rate sensors based on opposite-type infrared photoelectric cell for counting the dropping seeds; (b) a decimeter GPS receiver for acquiring planter position and operation speed; (c) a vehicle monitoring terminal based on ARM Cotex-m 4 core chip to acquire and process the whole-system data; (d) a touchscreen monitor to display the planter performance for the operator; and (e) a buzzer alarm to sound a warning when skip and double seeding happened. Taking the applicability, dependability and feasibility of the monitoring system into consideration, the opposite-type infrared photoelectric sensors were selected and their deployment strategies in the 6-port seed tube were analyzed. To decrease the average response time, a distributed information communication structure was adopted. In this information communication mode, collectors were designed for each individual sensor and communicated with sensors through two-wire CAN bus. A sensor together with the designed collector is called a sensor node, and each of them worked individually and took the responsibility for acquiring, processing, and transiting the on-going information. Laboratory test results showed that the random error distribution was approximately normal, and by liner analysis, the system observed value and the true value had as a liner relationship with coefficient of determination $R^{2}=0.9991$. Series of field tests showed that the seeding rate maximum relative error of the 6-port seed tube was $2.92 \%$, and the maximum root mean square error (RMSE) was about $1.64 \%$. The monitoring system, including sensor nodes, vehicle monitoring terminal and a touch-screen monitor, was proved to be dependable and stable with more than $14 \mathrm{~d}$ of continuous experiments in field.
\end{abstract}

Keywords: maize, precision planting, monitoring system, seeding rate, on-line assessment, CAN bus

DOI: $10.25165 /$ j.ijabe.20181104.3517

Citation: Yin Y X, Chen L P, Meng Z J, Li B, Luo C H, Fu W Q, et al. Design and test of precision seeding monitoring system for maize planter. Int J Agric \& Biol Eng, 2018; 11(4): 186-192.

\section{Introduction}

The management of inputs to crop production in agricultural field, especially the seeds, has been conducted by humans according to previous practices ${ }^{[1-3]}$. The advent of the integrated circuit technology and the associated development of embedded controls and sensing technologies permit maize planting to be made with greater specificity, precision, and accuracy. In other words, precision planting (PP) has been applied in agriculture for some years and is strongly advocated in China ${ }^{[4,5]}$. The results of PP are better seed spacing, better depth control and better root systems through adopting fresh technologies. PP improves the field

Receive date: 2017-06-24 Accepted date: 2017-12-19

Biographies: Yanxin Yin, PhD, Research Assistant, research interests: intelligent agricultural equipment, Email: yinyx@nercita.org.cn; Liping Chen, $\mathrm{PhD}$, Professor, research interests: intelligent agricultural equipment, Email: chenlp@nercita.org.cn; Bin Li, PhD, Associate Professor, research interests: intelligent agricultural equipment. Email: lib@nercita.org.cn; Changhai Luo, Master, research interests: measurement \& control technology in agriculture. Email: luoch@nercita.org.cn; Weiqiang Fu, Master, Associate Professor, research interests: measurement \& control technology in agriculture, Email: fuwq@nercita.org.cn; Hebo Mei, Master, research interests: measurement \& control technology in agriculture, Email: meihb@nercita.org.cn; Wuchang Qin, Master, research interests: measurement \& control technology in agriculture, Email: qinwc@nercita.org.cn.

*Corresponding author: Zhijun Meng, PhD, Research Fellow, research interests: intelligent agricultural equipment. Email: mengzj@nercita.org.cn. management from several perspectives. For example, it can help to minimize waste of seeds by eliminating the need for thinning in certain field operations, leading to more efficient, economical and talented agriculture ${ }^{[6-13]}$. There are many criteria required for placing seeds in PP, including seeding rate, seed spacing, seed meters and methodologies, positions within the furrow, planting depth, etc ${ }^{[14-18]}$.

The planter is a main farm implement for PP. Plant spacing uniformity and emergence rate are the most common characteristics used by producers to evaluate planter performance which has a huge impact on yield ${ }^{[19-21]}$. There are generally two ways to improve a planter's performance, one is to optimize the design of the planter, especially the mechanical structure of seed meters, another one is to real-time monitor the process of planting to diagnose planting problems (e.g., lost or double seed, seed tube stock, missing seeds, etc.). For the purposes of continuously monitoring the planting process, detecting problems at an early stage, and adjusting the operation parameters in real-time, the second way was a better choice ${ }^{[22-25]}$.

Some researches on the seed detection for precision planting were conducted in recent years. Ji et al. ${ }^{[26]}$ adopted reflection-type photoelectric cell to develop a monitoring system for working performance of no-tillage corn precision seeder. They also designed a self-cleaning device against dust. The system could count seeds with accuracy of $98.1 \%$ in simulated dusty condition. Zhou et al. ${ }^{[27]}$ studied the method of using a parallel-plate capacitor 
to detect corn seeds, and by laboratory test, the accuracy of seed counting was $94.6 \%$. He et al. ${ }^{[28]}$ designed a testing instrument for maize precision seed meter's performance detection with photoelectric seed sensor. The instrument could detect and calculate the qualified rate, missing rate, reseeding rate, variability of seed-space for seed meter. By comparative bench tests, the maximum error of counting was less than $2 \%$. Chen et al. ${ }^{[29]}$ used a high-speed camera to capture the picture of seeding process, and obtained seed number by image analysis. Tests under JPS- 12 bench indicated that the seeding qualified index was less than $1 \%$ and coefficient of variation was less than $3 \%$.

On analysis of the above mentioned researches, it was found that photoelectric cell and visual sensor are the two main types of sensors, which are sensitive to the shapes of the kernels dropping though the seed meters in bench-top laboratory tests. The visual sensor, however, was not the optimal choice for practical application because it was easily interfered by natural light, dust, dirty or pollen. Whereas, the photoelectric cell, mainly involving reflection-type and opposite-type, senses the mass of the kernels by a beam of light that are not sensitive to dust or other debris. Moreover, the photoelectric cell performs well in field tests owing to the simple structure, brief circuit and rapid response. In a wheat seeding monitoring system designed by Beijing Research Center of Intelligent Equipment for Agriculture ${ }^{[30]}$, a new opposite-type photoelectric sensor with reasonable allocation of photoelectric elements and analog output was designed to improve monitoring accuracy and reliability, and a hall sensor was used to convert quantities of wheel axis rotation velocity into pulse signals. The monitoring system was proved to be reliable and have a strong ability to anti-dusting and anti-vibration according to series of field tests. The monitoring accuracy for seed tube fault status was up to $98 \%$.

The aim of this work is to provide farmers a dependable tool to real-time monitoring and on-line assessment of the performance of maize planting.

\section{Materials and methods}

\subsection{System overall design}

In order to cope with the strict requirement of high accuracy, rapid response and strong reliability, a realistic system was proposed, which including: (a) opposite-type infrared photoelectric cells for counting the seeds; (b) a decimeter GPS receiver to acquire planter position and operation speed; (c) a vehicle monitoring terminal based on ARM Cotex-m4 core chip for acquiring and processing the data of the whole system; (d) a touchscreen monitor to show the planter performance for the operator; (e) a buzzer alarm device to warn the operator when skip and double seeds happened. As shown in Figure 1, the opposite-type infrared photoelectric sensors mounted on lower part of the 6-port seed tube; the GPS module installed on top of tractor cabin and buzzer based alarm device fixed in the cabin; the touchscreen monitor connected to the vehicle monitoring terminal via CAN bus.

To decrease the average response time, a distributed information communication structure was adopted. Micro-processor based collectors were developed, which had the capability of performing, processing and gathering sensory information of each sensor placed in specific detection. This collector was able to convert the collected signal (e.g., analog signal, pulse signal) to the corresponding physical quantity (e.g., operation speed, seeding quantity), and transfer these processed information to vehicle monitoring terminal by CAN bus. In order to reduce the electric signal transmission loss of the sensors, the collectors should be mounted close to sensors. The sensor together with the collector was called a sensor node, and each sensor node worked individually.

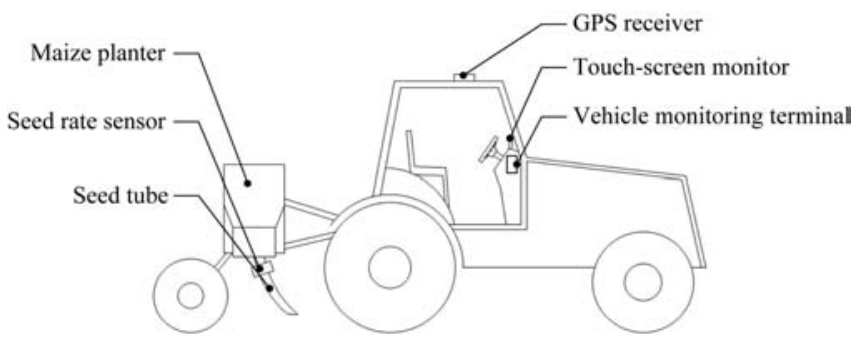

Figure 1 Overall layout of the monitoring system

The overall information communication system designed for monitoring planting process was shown in Figure 2. The working process is as follows: the sensor nodes (shown on the bottom of Figure 2) are responsible for acquiring, processing, and transiting the on-going information; the vehicle monitoring terminal (shown on the middle of Figure 2) is for handling and processing the data from each sensor node; the touchscreen monitor presents the parameters (e.g., seeding rate, operation speed and real-time location), and the alarm device gives an audible signal to the operator about a problem.

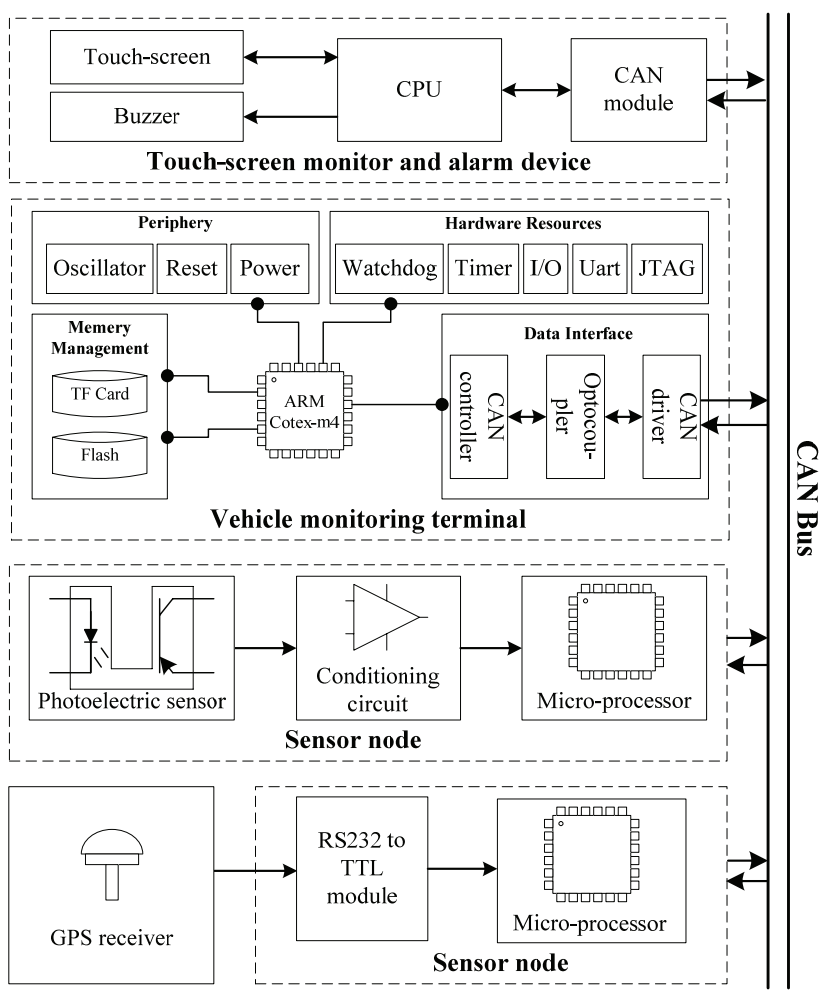

Figure 2 Overall structure design of the monitoring system

\subsection{Hardware components design}

In this paper, opposite-type infrared photoelectric cells were employed to detect maize kernels dropping through seed tubes. Taking the maize kernels' variation in dropping height into consideration, the number of cells to use and the layout of these cells in the tube cross-section to get all-size kernels (especially the micros) detected were studied.

\subsubsection{Layout optimization of photoelectric cells}

The schematic cross-section of the tube was depicted in Figure 3. Several pairs of photoelectric cells were set a circle around the inner wall of the tube. The parallel beam from the emitter to 
detector formed several detection zones. In the dead zone, the micro-kernel might be missed when dropping through. The relationship between dead zone and the seed is shown in Figure 3.

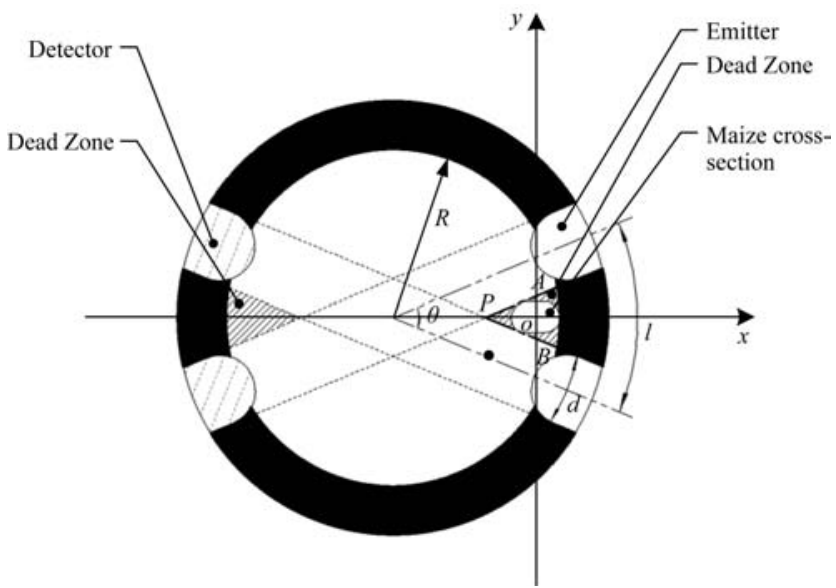

Figure 3 Schematic of tube cross-section and layout model of photoelectric sensor

To find out how many pairs of photoelectric cells should be set around the circle and how they were distributed, a geometric model was built and analyzed based on the maize motion attitude in tube. To make sure that each single seed could be detected when dropping through the dead zone, the smallest size of projection should be considered in the model. Generally, the shape of maize seed was considered as a ladder in front projection, a rectangle in side projection, and an ellipse in top projection, as shown in Figure 4 , among which, the size of top projection was the smallest. Therefore, the maize seed was considered as an ellipse in xoy coordinate, as shown in Figure 5.

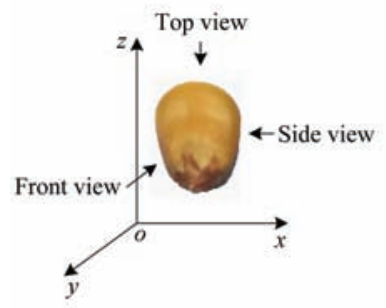

a

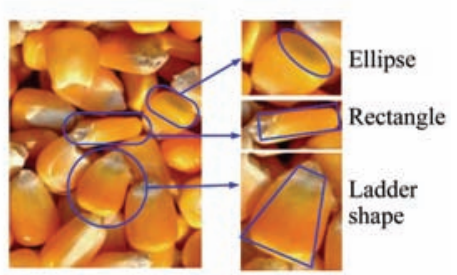

Figure 4 Maize seed's shape in different viewpoints

In Figure 5, the included angle between the two contiguous beams was $\theta$. The long axis and short axis of the ellipse were $a$ and $b$. The dead zone was an isosceles triangle $\triangle P A B$.

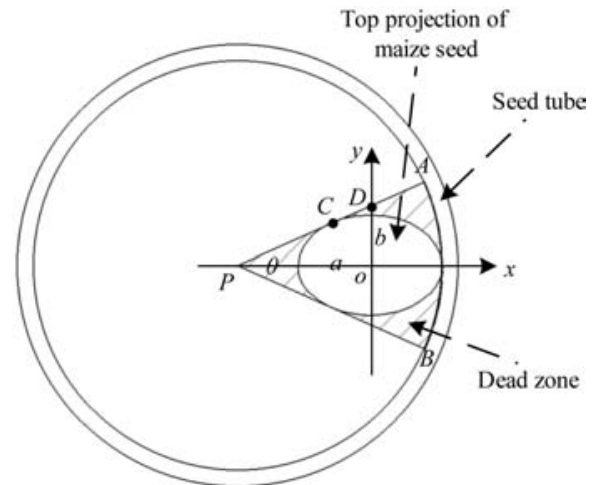

Figure 5 Relationship of dead zone and seed model in xoy coordinate

In the xoy coordinate, line $P A$ was circumscribed with the ellipse. Assuming the elliptical equation is

$$
\frac{x^{2}}{a^{2}}+\frac{y^{2}}{b^{2}}=1
$$

Line $P A$ is

$$
y=\tan \frac{\theta}{2} x+q
$$

where, $q$ was the intercept of $y$ axis.

According to geometric principle, the coordinate of point $P$ was

$$
\left(\frac{d}{2 \sin \frac{\theta}{2}}-R, 0\right)
$$

where, $d$ was the diameter of the parallel beam from photoelectric sensor; $R$ was the radius of seed tube.

The Equation (2) was

$$
y=\tan \frac{\theta}{2} x+R \tan \frac{\theta}{2}-\frac{d}{2 \cos \frac{\theta}{2}}
$$

Assuming point $C$ and $D$ were coincided, the following inequality would be obtained.

$$
2 R \cdot \sin \frac{\theta}{2}-2 a \cdot \cos \frac{\theta}{2} \leq d
$$

The number of photoelectric cell pairs adopted in this test was set as $\mathrm{n}$ and can be calculated by:

$$
n \leq \frac{\pi R}{d}
$$

Apparently, $n \theta=\pi$, the following equation would be obtained

$$
\theta \geq \frac{d}{R}
$$

In this research, the tube radius was $R=15 \mathrm{~mm} . \quad 500$ maize seeds were measured to obtain the minimum $a=8 \mathrm{~mm}$ and $b=5 \mathrm{~mm}$, respectively. Considering the Equations (5) and (7), the reasonable interval of $d$ and $\theta$ are shown in the shaded area in Figure 6.

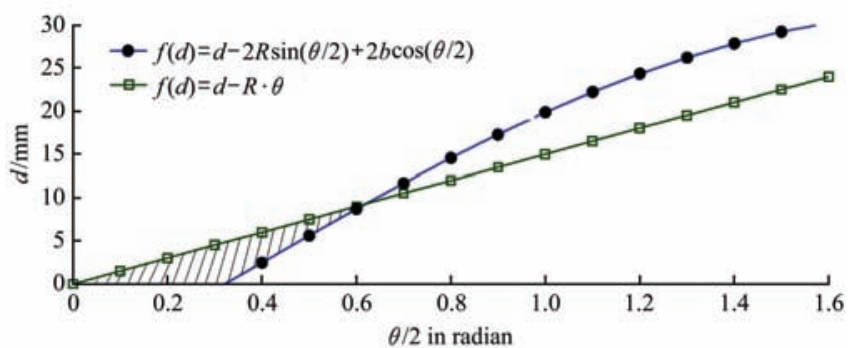

Figure 6 Reasonable interval of $d$ and $\theta$

To make dead zone as small as it can be, and interference of two contiguous pairs of photoelectric cells as slight as possible, the included angle was designed as $\theta=45^{\circ}$ ( 4 pairs of photoelectric sensors in total), and the diameter $(d)$ of the beam was set to $5 \mathrm{~mm}$ according to the relationship of $d$ and $\theta$.

\subsubsection{Circuit design and signal processing}

According to the detection principle of photoelectric cell, the detector should be turned off and low-voltage signal should be output when seed blocking between the emitter and detector, and high-voltage signal should be output after seed passed. Continuously sampling the detector's output voltage, theoretically, a dropout voltage could be detected. By the dropout voltage to judge whether a maize seed passes through the tube is the basic principle of this detection. The benefit is that the environment interference noise would be reduced.

The output voltages of the four detectors are $\boldsymbol{U}=\left[u_{1}, u_{2}, u_{3}, u_{4}\right]^{\mathrm{T}}$, 
$u_{i}(i=1,2,3,4)$, which can be obtained by the following equation:

$$
u_{i}=\beta_{i} I_{i} R
$$

where, $R$ was the resistant of the detector; $I_{i}$ was the base current and $\beta_{i}$ was the amplification factor.

According to Kirchhoff's current law (KCL), when the four detectors were connected as parallel circuit, the following equation was obtained

$$
U_{o} /(R / 4)=\sum_{i=1}^{4} \beta_{i} I_{i}
$$

where, $U_{o}$ was the output of the parallel circuit.

Thus,

$$
U_{o}=\frac{R}{4} \sum_{i=1}^{4} \beta_{i} I_{i}=\frac{u_{1}+u_{2}+u_{3}+u_{4}}{4}
$$

The Equation (10) indicated that the parallel circuit output voltage was the average of the four detectors' single output. In this research, the detecting circuit was designed as a parallel circuit.

To obtain the status of the seed tubes, an $\mathrm{AD}$ module of the microprocessor integrated in sensor node was used to continuously sample the output voltage of the circuit. In order to sample the output signal without distortion, sampling frequency should be analyzed. As shown in Figure 7, the kernel falls freely in the tube, and the initial dropping speed is $v_{0}$. According to Newton's law of motion

$$
\left\{\begin{array}{l}
v_{t}=v_{0}+g t \\
h_{0}=v_{0} t+\frac{1}{2} g t^{2}
\end{array}\right.
$$

where, $v_{t}$ is the seed dropping speed; $g$ is the acceleration of the free fall; $t$ is the elapsed time of kernel passing from the initial point to the sensor position; $h_{0}$ is the height between the initial point and the detecting sensor position.

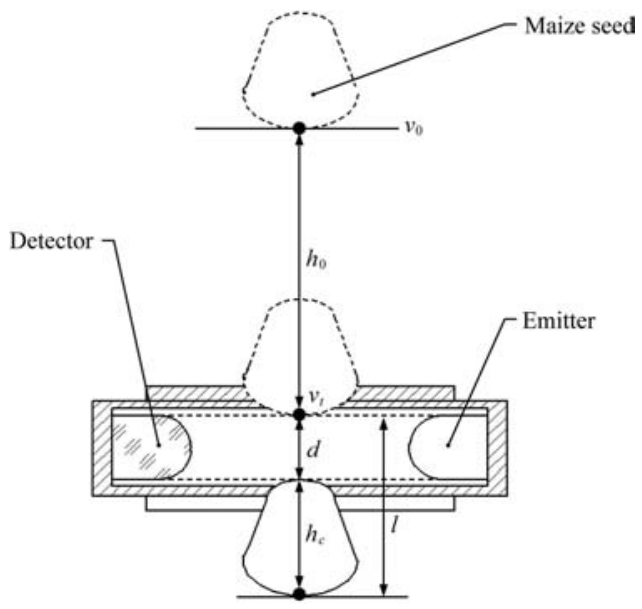

Figure 7 Dropping process of seed

The total distance travelled through detection area was

$$
l=d+h_{c}=v_{t} t_{x}+\frac{1}{2} g t_{x}^{2}
$$

where, $t_{x}$ is the elapse time of the seed passing through detection area. It is also the width of the signal pulse.

Set the initial speed as $v_{0}=0$. The following equation could be obtained according to Equations (11) and (12)

$$
l=d+h_{c}=\sqrt{2 g h_{0}} \cdot t_{x}+\frac{1}{2} g t_{x}^{2}
$$

In the research, the height $h_{0}=400 \mathrm{~mm}$, the minimum $h_{\mathrm{c}}$ was set as $8 \mathrm{~mm}$ by measuring 500 maize seeds, and $g=9.8 \mathrm{~m} / \mathrm{s}^{2}$.

Therefore, the signal pulse width was $t_{x} \approx 4.5 \mathrm{~ms}$, and the signal frequency was about $220 \mathrm{~Hz}$. According to Shannon sampling theorem, the sampling frequency should be at least twice the highest original signal frequency, or twice the highest analog frequency component. In practical application, to keep the sample data distortion less, the frequency should be much higher. Considering the complex working condition in field, the sampling frequency was set at $6000 \mathrm{~Hz}$. In a signal period, at least 20 sampling data could be obtained. To further weaken the unpredictable interference noise, the 20 sample data $S_{i}(i=1,2, \ldots, 20)$ were compared with the associated threshold value $S_{0}$. If $S_{i} \geq S_{0}$, it is considered the tube is empty, no maize kernel passing though. Otherwise, it is considered the maize kernel passed through the tube.

The probability of $S_{i} \geq S_{0}$ is made as follows:

$$
\begin{gathered}
p_{i}=\left\{\begin{array}{l}
0,\left(S_{i} \geq S_{0}\right) \\
1,\left(S_{i}<S_{0}\right)
\end{array}\right. \\
P\left(S_{i}<S_{0}\right)=\frac{1}{20} \sum_{i=1}^{20} p_{i}
\end{gathered}
$$

In the research, the threshold voltage $S_{0}$ was $30 \%$ of the high-level of the circuit output. And if $P\left(S_{i}<S_{0}\right) \geq 90 \%$, it is considered that the kernel was passing through the detection area. Figure 8 was showing the pulses triggered by two maize kernel passing through the sensor detection area in the tube.

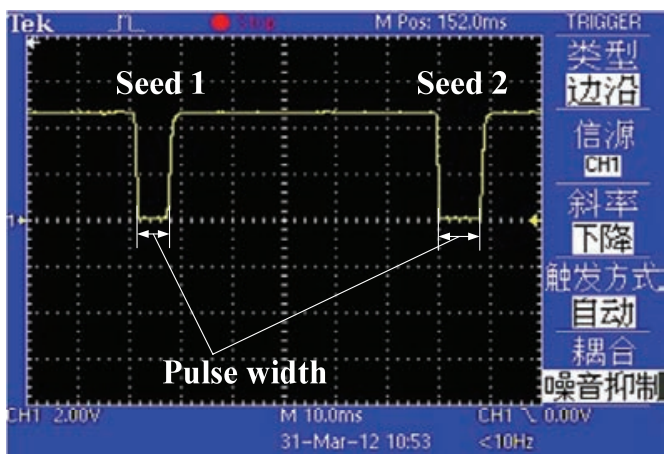

Figure 8 Pulses of two maize seed passing through the sensor detection area

\subsection{Software architecture}

The programs of the monitoring system was constructed by three-layer, taking the responsibilities of signal collection and pretreatment, further processing and transmission, data displaying and interactivity, respectively. The three-layer architecture was depicted in Figure 9.

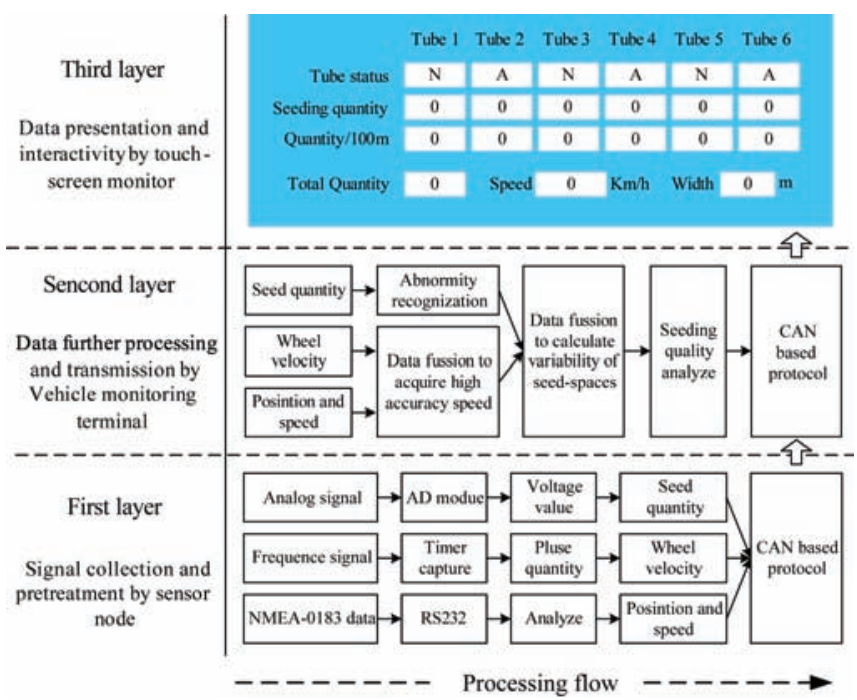

Figure 9 Architecture of three-layer framework 
In the first layer, analog signal, frequency signal and GPS data were collected by a microcontroller firstly, then filtered and analyzed to obtain the parameters of seeding rate, operation velocity and planter position. These parameters were transmitted to the vehicle monitoring terminal via CAN bus.

The second layer, running in vehicle monitoring terminal, was mainly focus on further data processing. In this layer, the parameters of seeding rate, operation velocity, together with the planter position, were used to conclude tube status, calculate the working distance and evaluate planting quality. Moreover, all the operational data was recorded in a flash before transmitted via CAN bus.

The third layer was set up in a touchscreen monitor, whose primary task was interactive data display. The software interface displayed the status of all the six tubes, seeding rate of each tube, seeding rate per $100 \mathrm{~m}$ of each tube, total rate, operation speed and width of the planter.

\subsection{Experiment}

\subsubsection{Calibration of seeding rate sensor}

To calibrate the seeding rate sensor and to assess the static performance of the system, series of tests were conducted using a self-designed test bench in laboratory. The test bench was shown in figure 10. The sensors were mounted on the tube below the seed discharge hole of the seeding meter. A motor mounted in the bench push the maize kernels discharging mechanism with constant rotate speed. A bag was set directly below the seed tube orifice to gather kernels. The kernel was counted and recorded manually.

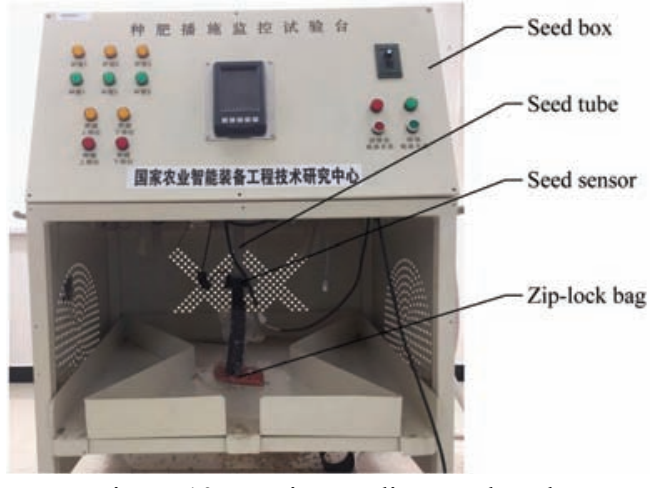

Figure 10 Maize seeding test bench

The tests were conducted in three groups according to the amount of seeds as follows: less than 1000 seeds, 1000-2000 seeds, and 2000-3000 seeds. It allows to reduce the random error and system errors by applying the necessary process in the successive seed dropping, as well as obtains a more realistic value for the monitoring system.

By analyzing a total of 117 seeding rate samples of the three groups, the absolute error distribution was shown in Figure 11.

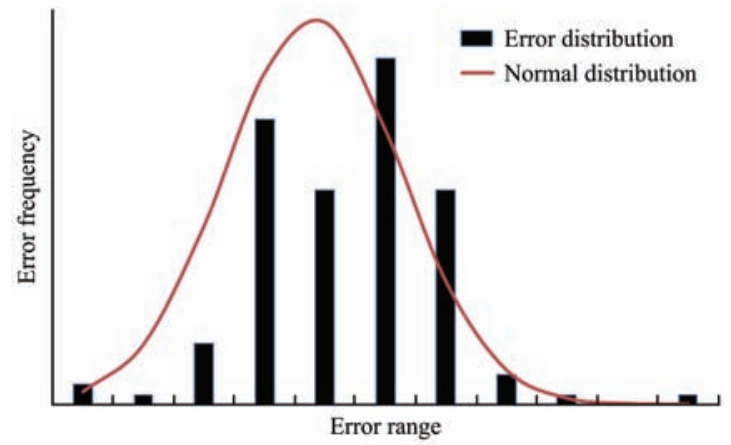

Figure 11 Error distribution of seeding rate monitoring
As shown in the Figure 11, the error distribution was approximately normal, which indicated that there was random error existing in the system. The random error was mainly due to the seed size, dropping attitude in the tube.

There was also system error existing in the monitoring system either as shown in Figure 12 by drawing all the data in a plane coordinator system. The horizontal axis indicated the seeding rate detected by the monitor system. The vertical axis indicated the real seeding rate counted manually. According to liner regression, the data presented as a liner relationship, and the $R^{2}$ was 0.9991 . Using the liner regression function to modify the test result could improve the accuracy of the seeding rate monitoring.

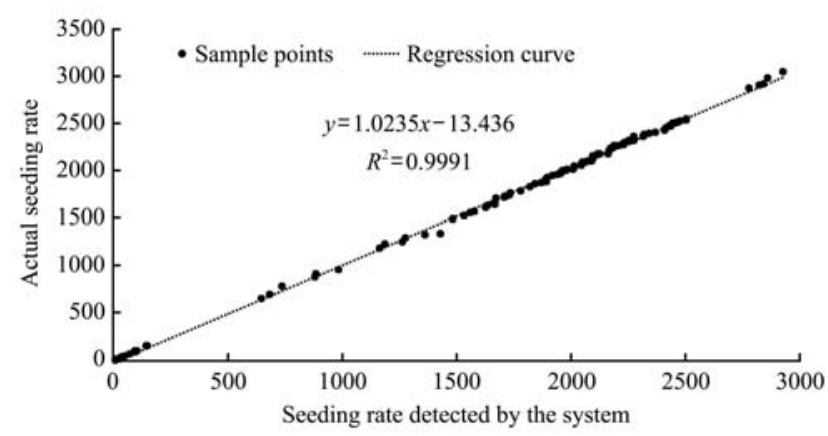

Figure 12 Calibrating curve for seeding sensor

\subsubsection{Field tests}

More than Fourteen groups of tests were carried out in National Experiment Station for Precision Agriculture, Beijing, China. A Haofeng maize planter with six tubes was selected. On each tube, a seeding rate sensor was mounted internally. At each tube orifice, a bag was strapped to collect kernels. In each group of tests, the actual seeding rate was counted and recorded manually according to the kernels in the bags. On top of the tractor cab, a GPS receiver was mounted. An overview of the experiment set-up, a tractor, seeding rate sensor and a GPS receiver was shown in Figure 13. In each test, this planter covered a distance of $400 \mathrm{~m}$, at speed of $3.5 \mathrm{~km} / \mathrm{h}$, while the seeding rate increased randomly by adjusting the seed-metering mechanism manually.

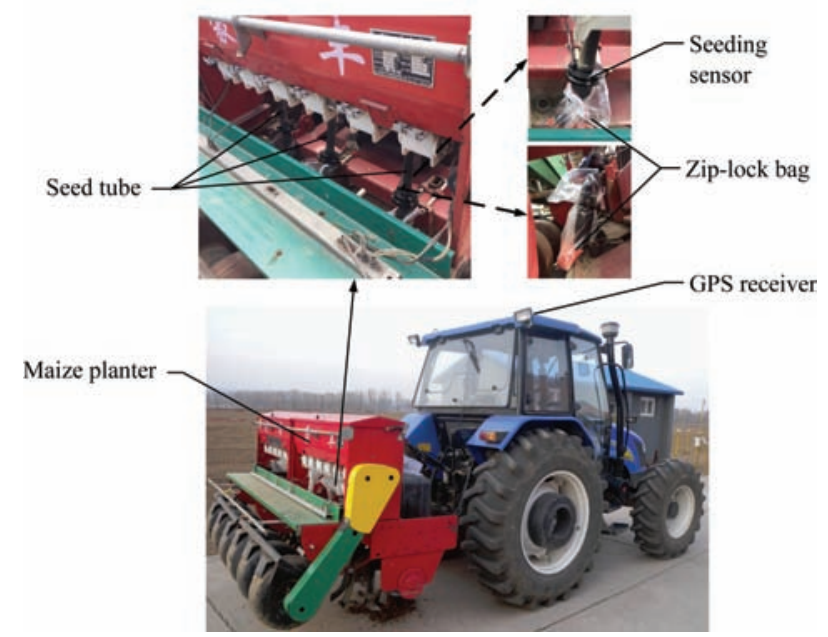

Figure 13 Overview of the experiment set-up

\section{Results and discussion}

The seeding rate of each tube from manual count and automated system test was shown in Figure 14. The horizontal axis was the group numbers of the tests. The vertical axis was seeding rate. The bar chart was the comparison between manual 
count seeding rate and automated system test results, and the curves showed the error between them.

The actual seeding rate increased as tests going on, as shown in the bar chart. Within a group of tests, however, the actual seeding rate of each tube was different. The reason was that the planter had a bad sowing uniformity between the tubes, and the monitoring system detected the uniformity correctly.

According to the error curves, the minimum and maximum relative errors of the six tubes were $-2.74 \%$ and $2.92 \%$, respectively. The root mean square error (RMSE) always reflects the precision and accuracy of the samples. According to the seeding rate RMSE of the six tubes in the fourteen groups of tests, the maximum RMSE was $1.64 \%$, and the minimum RMSE was $0.53 \%$, which indicated that the system had a high precision and accuracy in seeding rate monitoring.

From an overview of fourteen unrelated test groups, the error response of seeding system and the seeding rate has no significant regularity. Only one group results showed seeding error had an

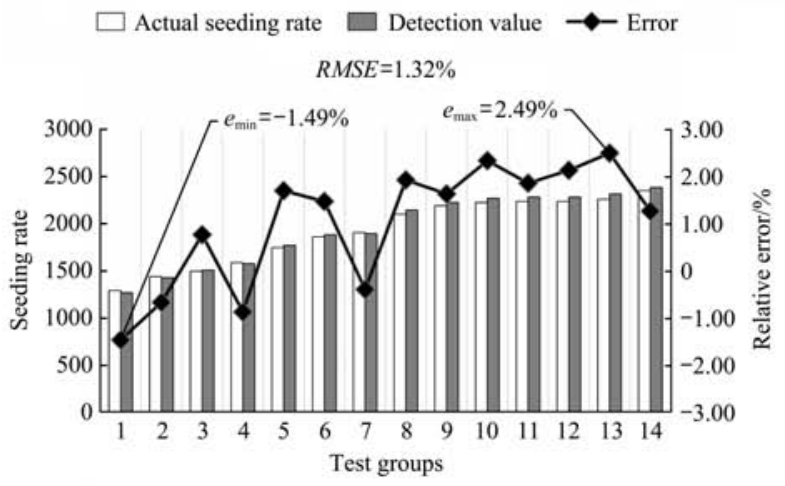

a. Test result of tube 1

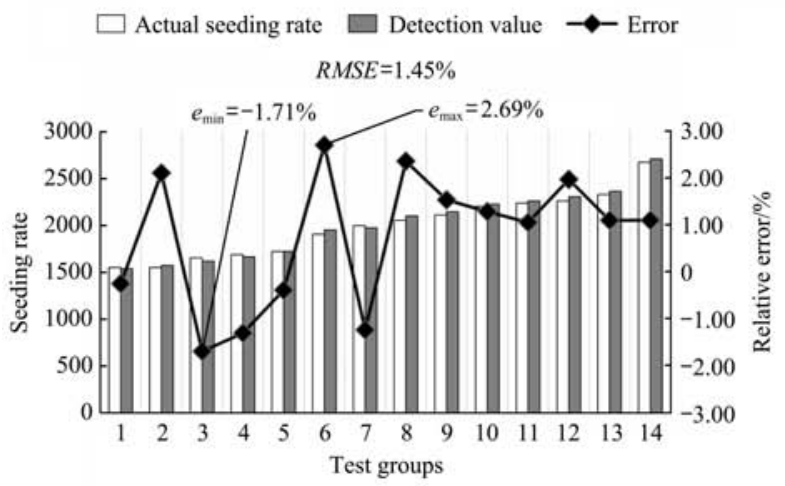

c. Test result of tube 3

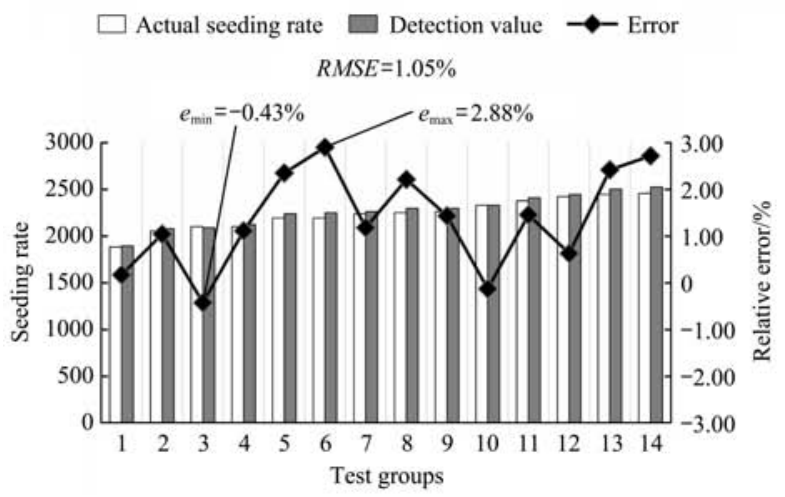

e. Test result of tube 5 increasing trend with seeding rate. With this clarification, we can get the conclusion that the seeding error was mainly caused by random error and had no direct relationship with systematic error. Two main factors contributed to the random error of seeding rate: 1) the corn seeds did not make an uninterrupted vertical drop due to the vibration of the seed tube and its sensors caused by working on uneven ground or driving up or down slopes, even if seed tubes remained almost vertical; 2) seeds of extreme minimum size slipped away from the detection area of seeding rate sensors.

To improve the performance and reduce the seeding error of the system, further study would be needed by: 1) optimizing the structure and parameters setting of seeding rate sensors by carrying out more experiments to improve the detection sensitivity; 2) designing special mechanical structure for seeding rate sensors deployment to against the effects of vibration on seed tubes; 3 ) further enhancing the system functionality by automatically measuring the seeded area and sowing distance and combing GIS to serve for precision agriculture.

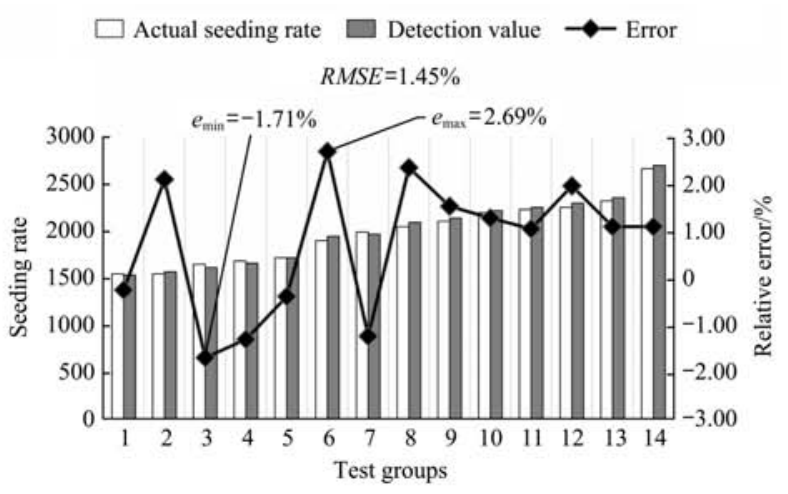

b. Test result of tube 2

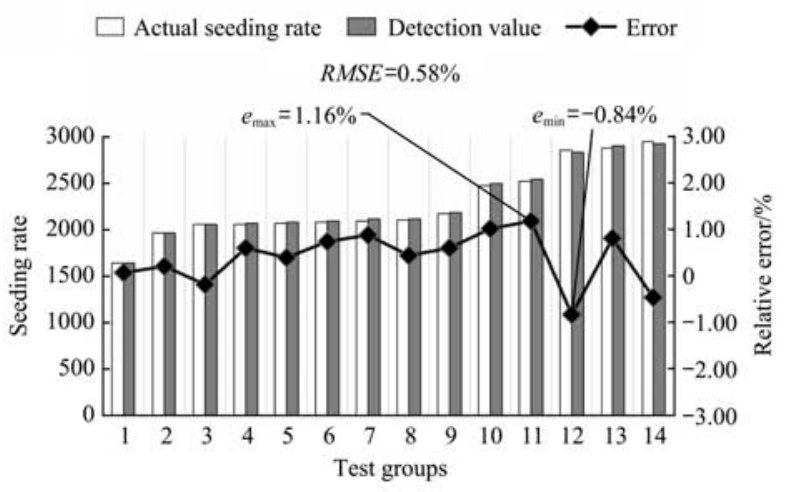

d. Test result of tube 4

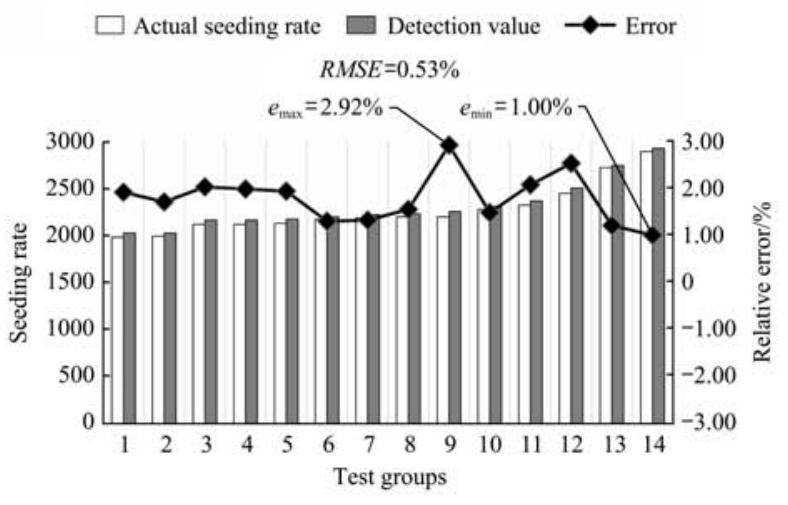

f. Test result of tube 6

Figure 14 Field test results of the sensors 


\section{Conclusions}

This study focused on a maize monitoring system for precision planting. Especially, the design and test method for seeding rate sensor based on photoelectric cell was explored. In the method, an opposite-type infrared photoelectric cells based seeding rate sensor was designed, and a calculation model for the number of photoelectric cells and their layout parameters were proposed for optimizing the structure of the sensor. From series tests both in laboratory and fields, the sensor had a high precision and accuracy. Of all the six tubes in field tests, the maximum error was $2.92 \%$, and the maximum RMSE was $1.64 \%$. This maize monitoring system provided a stable and dependable solution for real-time monitoring and online assessment of the performance of maize planting, and will significantly contribute to an improvement of precision planting operation. Furthermore, this system provided an experimental platform for designers to optimize the planter structural designs.

\section{Acknowledgements}

We acknowledge that this work was financially supported by the National Key Research and Development Program of China (2017YFD0700604, 2017YFD0700701), the Beijing Science \& Technology Plan Project (D161100003216001), the academy of science and technology innovation team program supported by Beijing Academy of Agriculture and Forestry (JNKYT201607).

\section{[References]}

[1] Young S L, Pierce F J. Automation: The future of weed control in cropping systems. Springer Netherlands, 2014; pp. 99-105.

[2] Shiva V. The Violence of the Green Revolution: Third World Agriculture, Ecology, and Politics, Zed Books Ltd., London, 2016; pp.231-257.

[3] Zhang Q. Precision agriculture technology for crop farming. CRC Press, 2015; pp.279-295.

[4] Zhang Z, Zhou X B, Chen Y H. Effects of irrigation and precision planting patterns on photosynthetic product of wheat. Crop Economics, Production \& Management, 2016; 108: 2322-2328.

[5] Qi J T, Jia H L, Li Y, Yu H, Liu X H, Lan Y B, et al. Design and test of fault monitoring system for corn precision planter. Int J Agric \& Biol Eng, 2015; 8(6): 13-19.

[6] Reddy P P. Sustainable intensification of crop production. Springer, 2016; pp. 143-154

[7] Thompson T A. Within-row spacing effect on individual corn plant yield. University of Illinois at Urbana-Champaign, 2013.

[8] Lindsey K N. Factors impacting corn establishment and the role of uniform stand establishment on yield. North Dakota State University of Agriculture and Applied Science, 2015.

[9] Staggenborg S A, Taylor R K, Maddux L D. Effect of planter speed and seed firmers on corn stand establishment. Applied Engineering in Agriculture, 2004; 20(5): 573-580.

[10] Singh T P. Farm Machinery. Prentice-Hall of India Pvt. Ltd, 2017; pp. 85-90.

[11] Zhang D X, Yang S D, Diao P S, Guo Z D, Song J L, Zhang X D. Design and experiment of side positive pressure seed metering device. Transactions of the CSAE, 2015; 31(Supp.1): 8-15. (in Chinese)

[12] Cui T, Han D D, Yin X W, Li K H, Xiao L L, Yang L, et al. Design and experiment of inside-filling air-blowing maize precision seed metering device. Transactions of the CSAE, 2017; 33(1): 8-15. (in Chinese)

[13] Li Y H, Meng P X, He K, Meng F H, Jiang M. Intelligent system for adjusting and controlling corn seeding depth. Transactions of the CSAM, 2016; 47(Supp.1): 62-68.

[14] Reis Â V, Forcellini F A. Functional Analysis in the evaluation of four concepts of planters. Ciência Rural, Santa Maria, 2002; 32(6): 969-975.

[15] Torres G M. Precision planting of maize. Oklahoma: Oklahoma State University Stillwater, 2011.

[16] Wang J W, Tang H, Wang Q, Zhou W C, Yang W P, Shen H G. Numerical simulation and experiment on seeding performance of pickup finger precision seed-metering device based on EDEM. Transactions of the CSAE, 2015; 31(21): 43-50. (in Chinese)

[17] Zhao J K, Jia H W, Guo M Z, Jiang X M, Qu W J, Wang G. Design and experiment of supported roll-cutting anti-blocking mechanism with for no-till planter. Transactions of the CSAE, 2014; 30(10): 18-25. (in Chinese)

[18] Yang Y L, Gu S, Li K, Liu K, Zhang Q, Zhong L X, et al. Parameters optimization of directing precision seeder for large cucurbitaceous seeds. Transactions of the CSAE, 2013; 29(13): 15-21. (in Chinese)

[19] Thompson T A. Within-row spacing effect on individual corn plant yield: Urbana, University of Illinois at Urbana-Champaign, 2013.

[20] Novak L K. Factors impacting corn establishment and the role of uniform stand establishment on yield. Fargo, North Dakota State University of Agriculture and Applied Science, 2015.

[21] Yang L, Yan B X, Cui T, Yu Y M, He X T, Liu Q W, et al. Global overview of research progress and development of precision maize planters. Int J Agric \& Biol Eng, 2016; 9(1): 9-26.

[22] Chu J, Lu H D, Xue J Q, Zhao M. Field experiment and effect of precision mechanical sowing of maize based on wide-narrow deep rotation and -no-tillage technology. Transactions of the CSAE, 2014; 30(14): 34-40. (in Chinese)

[23] Liu Z J, Liu L J, Yang X J, Zhao Z B, Liu X Q. Deign and experiment of no-till precision planter for corn. Transactions of the CSAE, 2016; 32(Supp.2): 1-6. (in Chinese)

[24] Huang D Y, Zhu L T, Jia H L, Yu T T, Yan J. Remote monitoring system for corn seeding quality based on GPS and GPRS. Transactions of the CSAE, 2016; 32(6): 162-169. (in Chinese)

[25] Zhang R, Cui T, Han D D, Zhang D X, Li K H, Wang Y X, et al. Design of depth-control unit with single-side gauge wheel for -no-tillage maize precision planter. Int J Agric \& Biol Eng, 2016; 9(6): 56-63.

[26] Ji C, Chen X G, Chen J C, Wang S G, Hao P L. Monitoring system for working performance of no-tillage corn precision seeder. Transactions of the CSAM, 2016; 47(8): 1-7.

[27] Zhou L M, Wang S M, Zhang X C, Yuan Y W, Zhang J N. Seed monitoring system for corn planter based on capacitance signal. Transactions of the CSAE, 2012; 28(13): 16-21. (in Chinese)

[28] He X T, Hao Y L, Zhao D Y, Zhang D X, Cui T, Yang L. Design and experiment of testing instrument for maize precision seed meter's performance detection. Transactions of the CSAM, 2016; 47(10): 19-23.

[29] Chen J, Bian J A, Li Y M, Zhao Z, Wang J L. Performance detection experiment of precision seed monitoring device based on high-speed camera system. Transactions of the CSAE, 2009; 25(9): 90-95. (in Chinese)

[30] Lu C Y, Fu W Q, Zhao C J, Mei H B, Meng Z J, Dong J J, et al. Design and experiment on real-time monitoring system of wheat seeding. Transactions of the CSAE, 2017; 33(2): 32-40. 